\title{
Case Report: Resolution of Hypoglossal Nerve Palsy Following Aspiration of a Branchial Cyst
}

\author{
Noora Althawadi $i^{*}$, Mohamed Alreefi ${ }^{1}$, Deepti Sinha ${ }^{1}$ and Susan Jawad ${ }^{2}$ \\ ${ }^{1}$ Head and Neck Department, University College London Hospital, United Kingdom \\ ${ }^{2}$ Radiology Department, University College London Hospital, United Kingdom
}

\begin{abstract}
Cranial nerve palsies caused directly by branchial cysts is a rare entity. We report a case of hypoglossal nerve palsy in a 47-year-old female with a right anterior neck mass presenting with symptoms of speech disturbances, altered tongue sensation and deviation on protrusion. An ultrasound scan showed a cystic lesion highly suspicious for a branchial cyst, which was confirmed with a head and neck MRI. An ultrasound guided aspiration of the cyst resulted in resolution of the nerve palsy in our patient. There have been cases reported in literature whereby the nerve palsies resolve following surgical excision of the branchial cyst.
\end{abstract}

\section{Keywords}

Branchial cyst, Hypoglossal nerve palsy, Aspiration

\section{Introduction}

Branchial cleft cysts are benign congenital anomalies that present as anterior neck masses either at birth or following an upper respiratory tract infection. They can arise from both the first and second branchial clefts, with the latter being more common, as a result of incomplete involution of the branchial cleft. The cyst itself is usually lined with stratified squamous epithelium [1-3]. Complications of branchial cysts include infections and abscess formation. Damage to nearby neurovascular structures typically occurs iatrogenically at the time of resection. Cranial nerve palsies caused directly by the cyst itself are a rare entity [4].

We report a case of hypoglossal nerve palsy caused by a branchial cyst, which completely resolved following aspiration.

\section{Case Report}

A 47-year-old female was referred for a right sided neck lump to the Head and Neck Surgery clinic as part of the two week wait pathway for head and neck cancers. A telephone consultation was made with the patient in view of the current COVID-19 pandemic. The patient gave a one-year history of a right sided neck lump which was progressively increasing in size, triggered by severe upper respiratory tract infection. The patient also felt at the time that her speech was affected, and her tongue was heavy on the same side. Her medical history was non-contributory apart from undergoing a right hemithyroidectomy many years ago for unknown pathology. An ultrasound scan of the neck performed was highly suspicious of a branchial cyst. The patient was referred to the radiology department for an MRI scan and an ultrasound guided fine needle aspiration of the neck as part of diagnostic workup.

The head and neck MRI showed a cystic lesion measuring $3 \times 3 \mathrm{~cm}$ in the right level 2 neck which had MR features compatible with a branchial cyst; well demarcated, homogenous lesion with high signal on $\mathrm{T} 2$ images $[5,6]$. A concomitant right hypoglossal nerve palsy, with no cause identified at the right hypoglossal canal or intracranial compartment was observed. The only other structural cause for the palsy was compression of the hypoglossal nerve at the level of the right posterior belly of digastric by the branchial cyst (Figure 1).

The cyst was then aspirated under ultrasound guidance and almost complete decompression was achieved (Figure 2). Cytopathological study of the cyst fluid showed a moderately cellular sample composed of haemosiderin laden macrophages in a background of blood and degenerate inflamma-

*Corresponding author: Dr. Noora Althawadi, Head and Neck Department, University College London Hospital, 250 Euston Road NW1 2PG, London, United Kingdom

Accepted: December 28, 2020

Published online: December 30, 2020

Citation: Althawadi N, Alreefi M, Sinha D, et al. (2020) Case Report: Resolution of Hypoglossal Nerve Palsy Following Aspiration of a Branchial Cyst. J Head Neck Surg 2(1):105-107 



Figure 1: (A) Coronal T2-w MRI showing a cystic structure in the right upper neck in keeping with a branchial cyst (arrow). The branchial cyst abuts and deviates the posterior belly of digastric at the expected anatomical position of the traversing right XII ${ }^{\text {th }} \mathrm{cranial}$ nerve (arrowheads); (B) Axial T2-W MRI showing a cystic structure in the right upper neck in keeping with a branchial cyst (arrow). The branchial cyst abuts and deviates the posterior belly of digastric at the expected anatomical position of the traversing right XII ${ }^{\text {th }} \mathrm{cranial}$ nerve (arrowhead). Note the laxity of the right side of the tongue that causes fullness at the right side of the tongue base (star); (C) Coronal T2-w MRI showing laxity and mildly increased T2-w signal at the right hemitongue in keeping with a right hypoglossal nerve palsy (arrow).

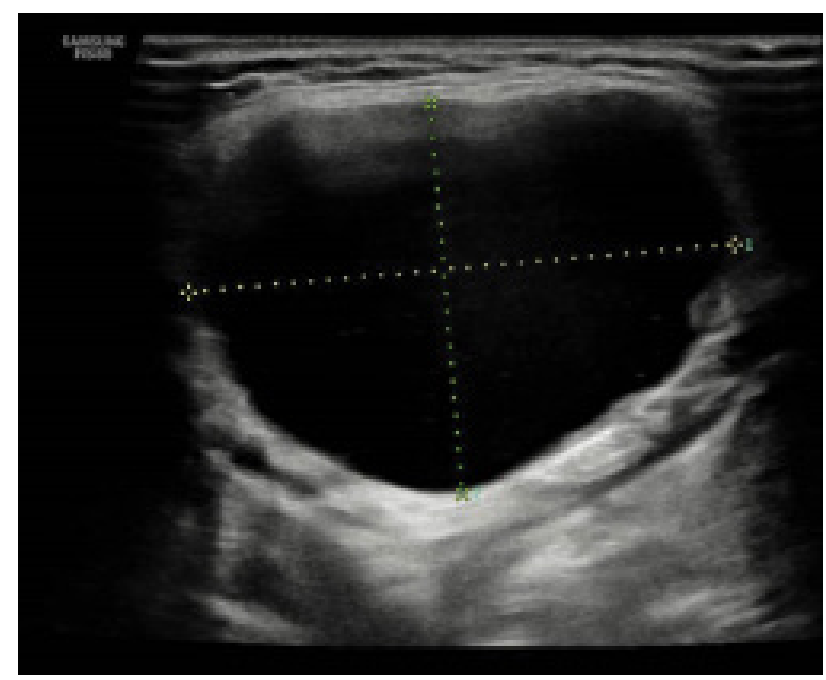

Figure 2: Ultrasound Scan of Neck showing $4 \mathrm{~cm} \times 2.9 \mathrm{~cm}$ lesion.

tory cells. No malignant cells were identified in the aspirate.

Following the aspiration, the patient reported return of normal speech and tongue sensation, along with resolution of her neck mass. She is awaiting surgical excision of right branchial cyst.

\section{Discussion}

Direct compression of the hypoglossal nerve by a branchial cyst is a rare entity. Damage to nearby neurovascular structures is typically seen following surgical extraction or in malignant lesions whereby invasion occurs. A review published in 2016 by the Mayo Clinic in the United States of
America looked into the etiology behind hypoglossal nerve palsies. Research has shown that the most common cause for cranial nerve XII palsy was (iatrogenic) postoperative (29.3\%) followed by idiopathic (15.1\%). Cystic lesions, which include branchial cleft cysts, only comprise $2.5 \%$ of hypoglossal nerve palsies [7].

In 2013, K Long, et al. described a case where a 10-yearold boy was intraoperatively found to have a branchial cyst completely encircling the hypoglossal nerve. Their patient had no pre-operative symptoms that would have suggested nerve involvement, as opposed to the patient we described in this case report [4]. In another case report published by S Mukherjee, et al. in 2012, reported iatrogenic hypoglossal nerve palsy in an 11-year-old boy following surgical excision of the branchial cyst [8].

Similar to our patient, there were two published case reports where a patient presented with both a branchial cyst and simultaneous hypoglossal nerve palsy. D Black, et al. reported a 20-year-old female who just like our patient underwent fine needle aspiration of the cyst prior to surgical excision. However, their patient's nerve palsy did not improve until formal surgical excision of the cyst has been done [9]. This also applies to the case series, with two cases, reported by $A$ Gatot, in 1991, where two patients presented with a branchial cleft cyst and manifestations of hypoglossal nerve palsy, but again only resolved following surgical intervention [10].

With regards to symptoms of hypoglossal nerve palsy, these may vary, dysarthria and tongue heaviness/swelling being the more common presentations $(28.3 \%$ and $22.3 \%$ respectively) [7]. These are also very typical of ischaemic and haemorrhagic strokes; a more sinister diagnosis that requires 
prompt neurological/neurosurgical intervention to prevent permanent sequela.

It appears that decompression of the nerve maybe achieved by minimally invasive methods such as ultrasound guided aspiration by a skilled head and neck radiologist. This may allow for resolution of symptoms and nerve recovery whilst patients await formal surgical excision of branchial cysts and may also prevent permanent nerve damage. This is especially useful in the current COVID pandemic.

\section{Conclusion}

Branchial cysts may rarely present as cranial nerve palsies, including hypoglossal nerve palsy. Thorough history and clinical examination is required to arrive at a differential diagnosis. Malignant etiology must be ruled out to properly manage such cases. Fine needle aspiration of a branchial cyst is required for cytological analysis but could also provide temporary symptomatic relief in context of cranial nerve palsies caused by direct compression from a branchial cyst.

\section{References}

1. Mitroi M, Dumitrescu D, Simionescu C, et al. (2008) Management of second branchial cleft anomalies. Rom J Morphol Embryol 49: 69-74.
2. Patel S, Bhatt $A$ (2019) Thyroglossal duct pathology and mimics. Insights Imaging 10: 12.

3. Prosser JD, Myer CM (2015) Branchial cleft anomalies and thymic cysts. Otolaryngol Clin North Am 48: 1-14.

4. Long K, Spears C, Kenady D (2013) Branchial cleft cyst encircling the hypoglossal nerve. Journal of Surgical Case Reports 2013: rjt068-rjt068.

5. Gaillard F (2020) Second branchial cleft cyst | Radiology Reference Article | Radiopaedia.org.

6. Shin J, Lee H, Kim S, et al. (2020) Parapharyngeal second branchial cyst manifesting as cranial nerve palsies: MR Findings. American Journal of Neuroradiology 22: 510-512.

7. Stino A, Smith B, Temkit M, et al. (2016) Hypoglossal nerve palsy: 245 cases. Muscle Nerve 54: 1050-1054.

8. Mukherjee S, Gowshami C, Salam A, et al. (2012) A case with unilateral hypoglossal nerve injury in branchial cyst surgery. $J$ Brachial Plex and Peripher Nerve Inj 7: 2.

9. Black D, McCombe A, Okpala N (2013) Hypoglossal nerve palsy in the presence of a branchial cyst. Journal of Case Reports in Medicine 1: 1-3.

10. Gatot A, Tovi F, Fliss D, et al. (1991) Branchial cleft cyst manifesting as hypoglossal nerve palsy. Head Neck 13: 249-250.

DOI: $10.36959 / 605 / 550$ 